\title{
Temperature Variations of Forced Convection in Porous Media for Heating and Cooling Processes: Internal Heating Effect of Viscous Dissipation
}

\author{
Y.-M. Hung • C. P. Tso
}

Published online: 6 August 2008

(C) Springer Science+Business Media B.V. 2008

\section{Erratum to: Transp Porous Med DOI 10.1007/s11242-008-9226-8}

We would like to correct the errors that appeared at the press stage. In Section 2 [line \#18 in the paragraph containing Eq. (9)], the equation $\Phi=(\mu \vec{V} / k) \cdot \vec{V}+\mu \nabla^{2} \vec{V}$ should read as $\Phi=(\mu \vec{V} / k) \cdot \vec{V}+\frac{1}{2} \mu_{\text {eff }} \sum_{i} \sum_{j}\left(\partial v_{i} / \partial x_{j}+\partial v_{j} / \partial x_{i}\right)^{2}$, with $v_{i}$ the velocity components and $x_{i}$ the Cartesian coordinates.

Also, there are errors in Eq. (22) and Eq. (27). The correct form of Eq. (22) should read as

$$
\theta(Y)=\left(C_{1}^{\prime}+C_{2}^{\prime}\right) Y^{2}+C_{3}^{\prime} \cosh ^{2} \alpha Y+C_{4}^{\prime} \cosh \alpha Y+C_{5}^{\prime} .
$$

The correct form of Eq. (27) is

$$
\begin{aligned}
\mathrm{Br}_{\mathrm{c}}^{\prime}= & 4 \mathrm{Da}(\alpha \cosh \alpha-\sinh \alpha)\left[2 \alpha\left(\alpha^{2}-6\right) \cosh ^{2} \alpha+15 \sinh \alpha \cosh \alpha-3 \alpha\right] / \\
& \left\{\alpha\left[\alpha \cosh \alpha\left(4 \alpha^{2}-39\right)+\alpha\left(8 \alpha^{2}-66\right) \cosh ^{3} \alpha+\left(89 \cosh ^{2} \alpha+16\right) \sinh \alpha\right]\right. \\
& \left.-4 \psi(\alpha \cosh \alpha-\sinh \alpha)\left[2 \alpha\left(\alpha^{2}-6\right) \cosh ^{2} \alpha+15 \sinh \alpha \cosh \alpha-3 \alpha\right]\right\} .
\end{aligned}
$$

The online version of the original article can be found under doi:10.1007/s11242-008-9226-8.

Y.-M. Hung $(\bowtie) \cdot$ C. P. Tso

Faculty of Engineering and Technology, Multimedia University, 75450 Melaka, Malaysia e-mail: ymhung@mmu.edu.my

C. P. Tso

e-mail: cptso@mmu.edu.my 